\title{
ONTOLOGIA NA GESTÃO DE CONHECIMENTO JURÍDICO
}

\author{
Felipe Mozart de Santana Nascimento \\ Universidade Federal de Pernambuco \\ mozart.felipe@gmail.com
}

\author{
Fabio Assis Pinho \\ Universidade Federal de Pernambuco \\ fabiopinho@ufpe.br
}

\begin{abstract}
Resumo
Objetiva identificar a contribuição do uso de ontologias na gestão do conhecimento jurídico. A temática aqui desenvolvida é de forte relevância para os estudos de Organização e Representação do Conhecimento pela Ciência da Informação, principalmente por considerar a ontologia como um dos Sistemas de Organização do Conhecimento que buscam melhorias nos processos de tratamento da informação. Quanto aos aspectos metodológicos esta pesquisa é classificada quanto aos meios, como uma pesquisa exploratória e bibliográfica; quanto aos fins, esta pesquisa se classifica como uma pesquisa de inovação ou pesquisa tecnológica; quanto a abordagem do problema, esta pesquisa é considerada como uma pesquisa qualitativa na justificativa de analisar o sistema de ontologias e sua empregabilidade nas estâncias estratégicas de inteligência de negócios, em domínio controlado. Os resultados cooperam para representar, por meio de questões de competência, como as ontologias contribuem para o processo de gerenciamento do conhecimento.
\end{abstract}

Palavras-chave: Gestão do Conhecimento. Sistema de Organização do Conhecimento. Ontologia.

\section{ONTOLOGY IN THE MANAGEMENT OF KNOWLEDGE LEGAL}

\begin{abstract}
This objective research is identify the contribution of the use of ontologies in the management of knowledge legal. The research is of great relevance to the studies of Knowledge Organization and Representation by Information Science, mainly considering ontology as one of the Knowledge Organization Systems that seek improvements in the processes of information processing. As for the methodological aspects, this research is classified, as for the means, as an exploratory and bibliographical research; for purposes, this research is classified as a research of innovation or technological research; as far as the approach to the problem is concerned, this research is considered as a qualitative research in the justification of analyzing the ontology system and its employability in the strategic offices of business intelligence in a controlled domain. The results cooperate to represent, through questions of competence, how ontologies contribute to the knowledge management process.
\end{abstract}

Keyword: Knowledge Management. Knowledge Organization System. Ontology.

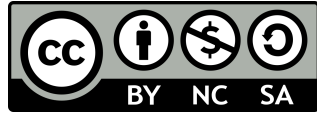




\section{INTRODUÇÃO}

Percebida como um elemento essencial nas organizações, a informação é um ativo gerado em todas as etapas de produção e, nelas, perpassa todas as cadeias de valor. Isso é corroborado nos estudos de Porter (1992) ao considerar que as atividades das cadeias de valor de qualquer organização criam e utilizam informações, além de apontar as dimensões de impactos que são percebidas por meio dos sistemas de informação gerencial (SIG) sobre a concorrência e os aspectos estratégicos competitivos.

Neste sentido, as organizações enfrentam uma conjuntura que reflete nos quesitos de sobrevivência mercadológica. Integram esta conjuntura as questões competitivas que contemplam novas práticas de gestão de informações. Percebe-se, então, que organizações vêm aplicando investimentos em pesquisas que têm como objetivo a adesão de novas práticas de Organização e Representação do Conhecimento (ORC) e que essas práticas possam resultar nas resoluções de necessidades informacionais e no apoio à tomada de decisão.

Destarte, esta pesquisa tem como justificativa a exploração das dimensões interdisciplinares que dialogam com a Ciência da Informação para as atividades de ORC, no caso desta pesquisa a dimensão jurídica pela natureza da informação que se é trabalhada e a dimensão administrativa por considerar os elementos resultantes como fatores estratégicos para tomada de decisão por meio do uso de ontologias.

Na perspectiva da ORC, o sistema de ontologia tem recebido atenção às potencialidades advindas de seu uso, sendo capaz de modelar conhecimentos por meio das especificações formais apresentadas e pela gestão de conhecimento realizada na área de domínio para a qual foi desenvolvida. Assim, questiona-se: de que forma o uso do sistema de ontologia auxilia gestão do conhecimento jurídico?

O uso de ontologia torna-se fundamental para o processo de desenvolvimento do conhecimento informacional por meio dos robôs de busca e está orientado pela Ciência da Computação e na Ciência da Informação como um caminho de buscas inteligentes e mais próxima a necessidade cognitiva do usuário (FUSCO; MUCHERONI; CONEGLIAN, 2017). Logo, apresenta-se como objetivo desta pesquisa identificar a contribuição do uso de ontologias na gestão do conhecimento jurídico.

Compreendido o contexto acima, a presente pesquisa visa identificar a contribuição do uso de ontologias na gestão do conhecimento jurídico a partir de buscas em um sistema de recuperação da informação. 


\section{ONTOLOGIA COMO SISTEMA DE ORGANIZAÇÃO DO CONHECIMENTO}

Os Sistemas de Organização do Conhecimento (SOCs) são considerados como instrumentos para representação do conhecimento e são utilizados na Ciência da Informação (CI) com o objetivo de organizar e recuperar informações, definem Lima e Maculan (2017, 61). Para as autoras é fundamental para a CI compreender como esses sistemas são construídos, principalmente na questão da ORC.

De acordo com Moreiro González (2011, p. 41) os Sistemas de Organização do Conhecimento:

- Permitem a categorização de recursos com esquemas de organização definidos, a fim de facilitar o processo de recuperação de informação;

- Permitem ao usuário a interpretação das estruturas de conhecimentos de maneira organizada;

- Contribuem para melhorias na eficiência dos serviços de informação, considerando também as soluções desenvolvidas para gerenciamento de informações;

- Buscam a terminologia específica das organizações. Neste ponto consideramse, para esta pesquisa, a terminologia empregada no domínio em estudo, ou seja, o domínio jurídico.

- Provêm do trabalho cooperativo no âmbito profissional. Aqui deve-se considerar os aspectos interdisciplinares empregados para desenvolvimento de estratégias organizacionais.

Temos como exemplos dos Sistemas de Organização do Conhecimento: taxonomias, tesauros e ontologias.

A taxonomia como SOC trata-se de uma lista de termos preferenciais com estruturas hierárquicas. Serve para ordenação de informações em um contexto hierárquico utilizando a relação pai-filho e são consideradas como estruturas classificatórias que servem como instrumentos para organização e recuperação da informação manifestando-se como mapas conceituais (MOREIRO GONZÁLEZ, 2011).

O tesauro pode ser considerado como "um instrumento de controle terminológico eficaz para a organização do conhecimento e importante ferramenta no tratamento e recuperação da informação" e são estruturas regidas por normas internacionais, 
como a ISO 2788 (1986), a ISO 5964 (1985) e a ANSI/NISO Z39.19-2003 (CARLAN, 2010, p. 40).

A ontologia recebe forte influência de diversas áreas do conhecimento, como a Filosofia e Ciência da Computação. Contudo, na Ciência da Informação, o sistema de ontologia é estudado como um "instrumento de representação do conhecimento, em geral, como um artefato computacional [...] que descreve formalmente termos e relações que podem existir entre eles dentro de determinado domínio" (LIMA; MACULAN, 2017, p. 65).

Considerada como a conceituação formal e explícita de um dado domínio (ou uma representação deste), a ontologia pode realizar operações em diferentes aplicações de softwares, como apresenta Moreiro González (2011), por exemplo: (I) os conceitos utilizados para a descrição servem como vocabulário comum, isso satisfaz a comunicação e interoperabilidade entre bancos de dados e outros recursos e (II) implicam na construção de sentidos nos resultados processados pelos motores de buscas.

O sistema de ontologia é construído por meio de diferentes mecanismos e fases de construção. Contudo, percebemos nos estudos que tratam sobre este sistema que não existe uma metodologia única para ser utilizada, visto que o uso de determina metodologia se afirma em seu grau de aplicabilidade por meio das fases de avaliação de uso do sistema.

Temos como razões para criação deste sistema ( $a$ ) o compartilhamento do entendimento comum das informações entre pessoas e sistemas, $(b)$ permissão de reutilizar o conhecimento de um domínio, $(c)$ explicitação de suposições de um domínio, $(d)$ separação do conhecimento sobre um determinado domínio do conhecimento operacional e (e) a análise de conhecimento de um domínio (MOREIRO GONZÁLEZ, 2011).

Para Moreiro González, devem ser levados em consideração alguns critérios principais adotados no desenvolvimento de ontologias, como os critérios clássicos no processo de recuperação da informação de exaustividade e precisão; deve ser considerado a análise taxonômica quanto a estrutura em que se apresenta o conhecimento, ou seja, é preciso realizar uma análise da estrutura conceitual da ontologia, a fim de observar eventuais erros de exaustividades e ou qualquer tipo de restrição.

Os elementos básicos para construção de uma ontologia estão apresentados em Ramalho (2010), onde considera os seguintes componentes: as classes e subclasses (onde agrupam um conjunto de elementos do mundo real, considera-se neste elemento o sistema taxonomia), as propriedades descritivas (onde descrevem as características das classes), as propriedades relacionais (onde tratam dos relacionamentos entres as classes), as instâncias 
(indicam os valores das classes e classes, considerando suas características relacionamentos e restrições), valores (atribuem valores às propriedades descritivas) e regras e axiomas.

Os dois últimos elementos retratados acima, conforme Ramalho (2010, p. 38) são considerados como "enunciados lógicos que possibilitam impor condições como tipos de valores aceitos, descrevendo formalmente as regras da ontologia e possibilitando a realização de inferências automáticas a partir de informações que não necessariamente foram explicitadas no domínio". É neste sentido que nos orientamos a seção a seguir, em que apresenta a identificação da contribuição do uso deste sistema na gestão de conhecimento jurídico.

\section{ONTOLOGIA NA GESTÃO DE CONHECIMENTO JURÍDICO}

A CI possui um interesse tradicional de se concentrar nos estudos voltados à documentação e nos registros de conhecimento. Percebe-se que a modelização dos domínios do conhecimento é considerada como um dos desafios a serem superados pela ciência em questão. Nesse sentido, a ideia de que recursos tecnológicos possam desenvolver atividades semelhantes ao cérebro humano, surgiram os estudos da Inteligência Artificial por meio da simulação de processos inteligentes e a aplicação de lógicas (DIAS; ALMEIDA; SOUZA, 2017).

Em consideração as organizações que exercem suas atividades em ambientes digitais, tais como departamentos e grandes escritórios jurídicos, pode-se apontar, de acordo com Fusco, Mucheroni e Coneglian (2017) como um dos grandes desafios: promover a integração das tecnologias na tentativa de suprir as necessidades dos processos de geração, acesso e controle de informações, assim as organizações se inserem em um cenário que exige uma evolução significativa dos processos de gestão e construção de conhecimentos.

Roberto Cerqueira, no desenvolvimento da pesquisa "Método de Modelagem DomínioOntológica do Direito Positivo Brasileiro”, em 2007, propôs um diagrama baseado no domínio em estudo, onde insere na base de conhecimento o conteúdo conceitual e o valor das leis emanadas do Poder Legislativo e que são, posteriormente, acessados por aplicativos. Esses aplicativos são capazes de recriar o documento original, ou seja, a lei e que podem acrescentar comentários que explicitam a informação implícita no formato original. Esse diagrama está representado a seguir: 
Figura 1: Exemplo de aplicação de sistema de representação de conhecimento baseado em ontologias (SRCO) na área jurídica.

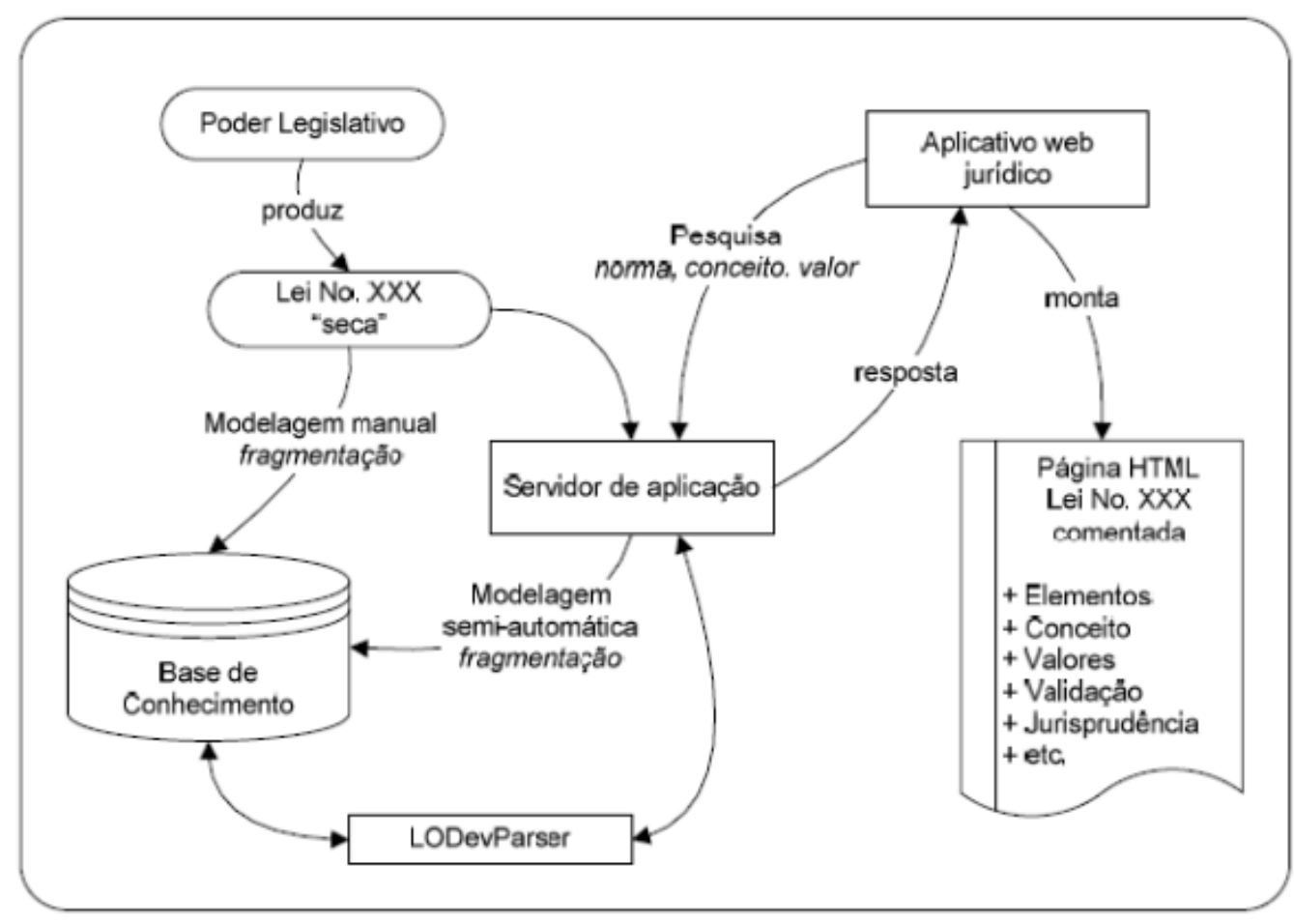

Fonte: Cerqueira, 2007, p. 41.

Conforme percebido na Figura 1, é possível a projeção de um sistema que permite além da navegação livre entre os documentos legais ou sua visualização por critérios textuais, a satisfação de indagações (CERQUEIRA, 2007).

Em consideração à satisfação de indagações por operadores do Direito, apontam-se como simulações as seguintes questões de competências, inferidas em um sistema de busca que utiliza ontologia em seu mecanismo de pesquisa: quais os assuntos que tiveram decisões menos favoráveis? Qual a quantidade de processos distribuídos no Estado de Pernambuco? Quais as comarcas que mais distribuem ações mais favoráveis sobre telefonia no Estado de Santa Cantarina? Quantas ações foram distribuídas desde o ano 2016 versam sobre processos trabalhistas no Estado de São Paulo?

As Figuras de 2 a 6, a seguir, baseadas nos dados do Conselho Nacional Justiça (2017), representam uma série de buscas que podem ser feitas em um sistema de recuperação de informação configurados em ontologias e que, por sua vez, são capazes de gerar novos insumos e valores informacionais aos tomadores de decisão como, por exemplo os operadores do Direito. 
Figura 2: Representação de busca baseada na questão de competência: 'Como está estruturado o primeiro grau do Poder Judiciário?'.

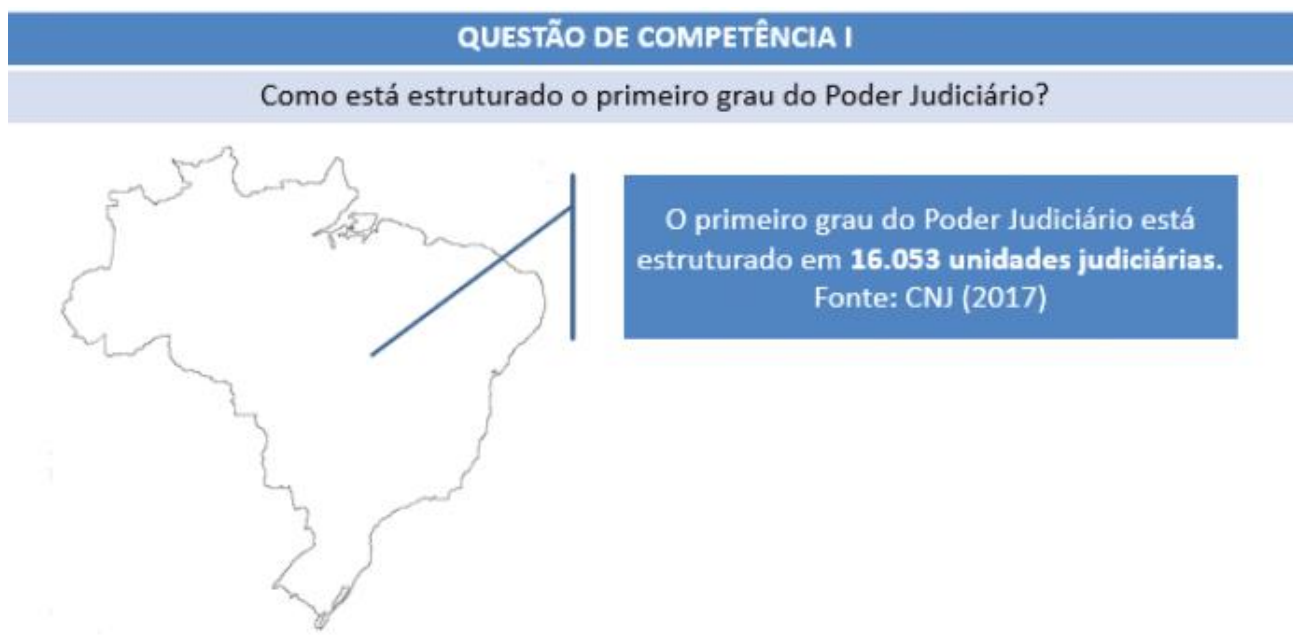

Fonte: CNJ, 2017.

Figura 3: Representação de busca baseada na questão de competência: 'Qual o número de unidades judiciárias de primeiro grau por ramo de justiça?'.

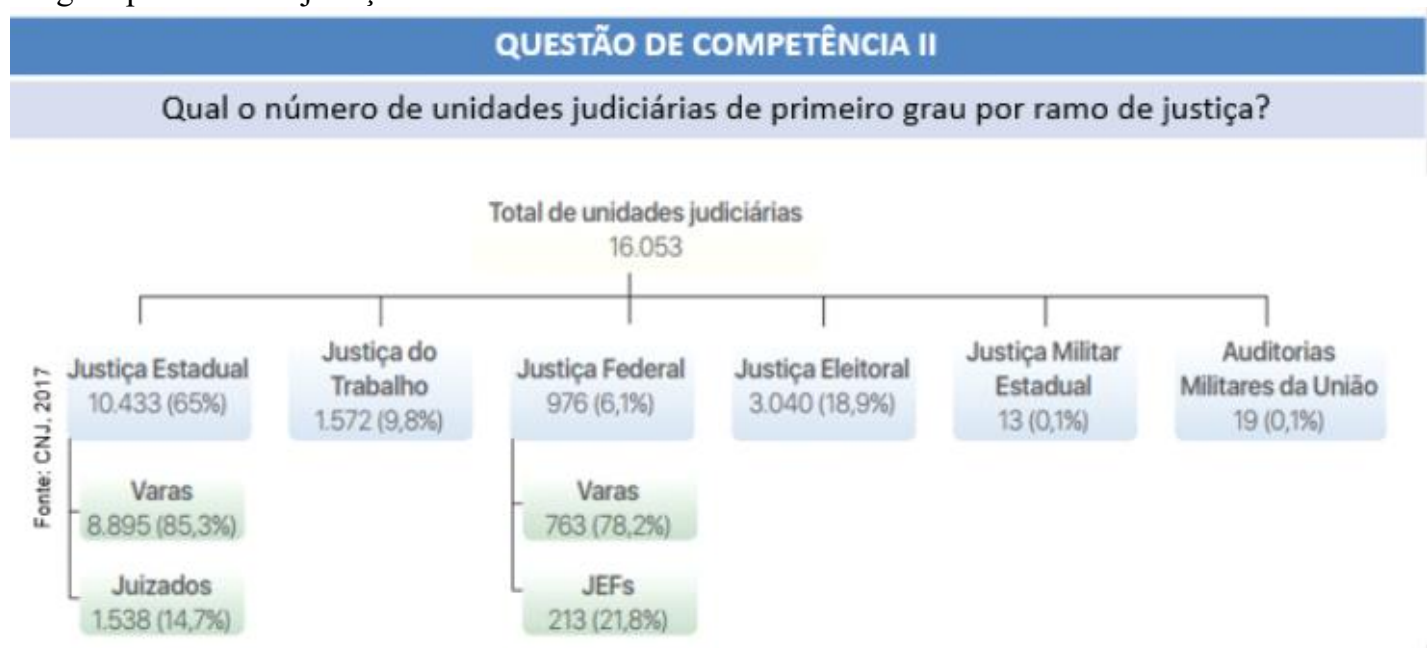

Fonte: CNJ, 2017. 
Figura 4: Representação de busca baseada na questão de competência: 'Qual o índice de unidades judiciárias de primeiro grau da Justiça Estadual por competência no ano de 2016?'.

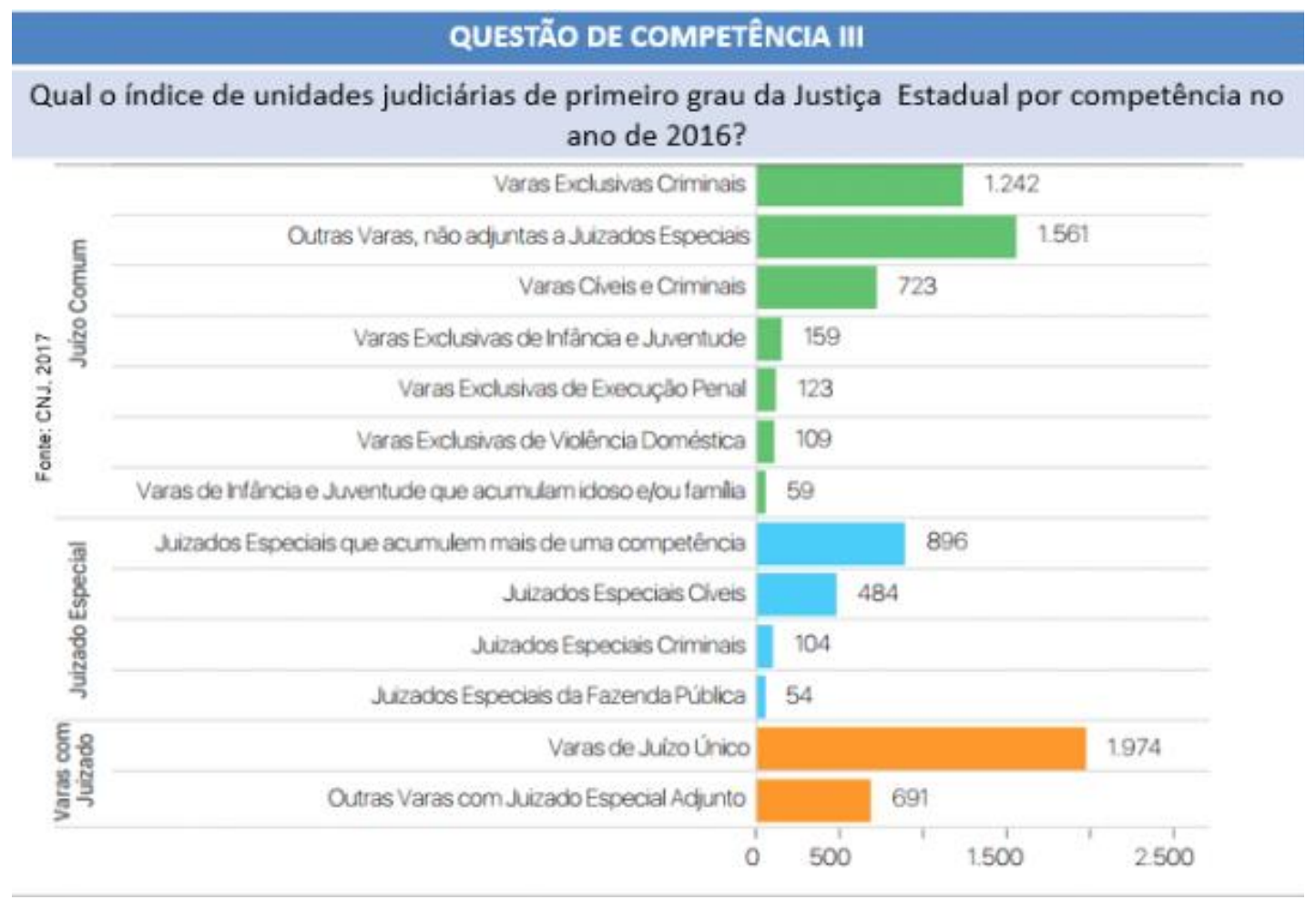

Fonte: CNJ, 2017.

Figura 5: Representação de busca baseada na questão de competência: 'Qual o índice de novos casos por ramo de justiça?'.

\section{QUESTẢO DE COMPETÊNCIA IV}

Qual o índice de novos casos por ramo de justiça?

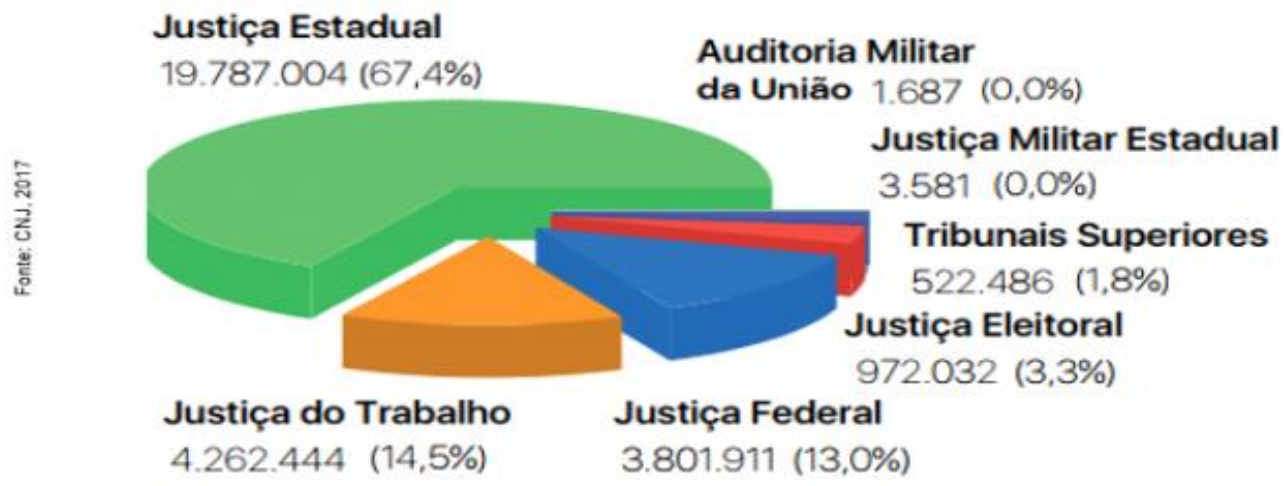

Fonte: CNJ, 2017. 
Figura 6: Representação de busca baseada na questão de competência: 'Quais os assuntos mais recorrentes na justiça do trabalho?'.

\begin{tabular}{|c|c|c|}
\hline \multicolumn{3}{|c|}{ QUESTÃO DE COMPETÊNCIA V } \\
\hline \multicolumn{3}{|c|}{ Quais os assuntos mais recorrentes na justiça do trabalho? } \\
\hline \multirow{5}{*}{ 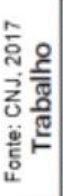 } & 1. DIREITO DO TRABALHO-Rescisäo do Contrato de Trabaho/Nerbas Rescisórias & $5.847 .967(11,51 \%)$ \\
\hline & 2. DIREITO DO TRABALHO-Responsablidade ONi do Empregador/indenizaçao por Dano Moral & $833.466(1,64 \%)$ \\
\hline & 3. DIREITO DO TRABALHO -Remuneraço, Verbas indenizatórias e Benesicios/Salärio / Diferençą Salarial & $636.148(1,25 \%)$ \\
\hline & 4. DIREITO DO TRABALHO-Rescisäo do Contrato de Trabaho/seguro Desemprego & $538.757(1,06 \%)$ \\
\hline & 5. DIREITO DO TRABALHO-Remuneraçäo, Verbas indenizatórias e Beneficios/Adicional & $375.092(0,74 \%)$ \\
\hline
\end{tabular}

Fonte: CNJ, 2017.

As questões de competências podem auxiliar no processo de melhoria dos sistemas, pois segundo Cerqueira (2007, p. 39): “[...] a melhoria significativa no suporte às tarefas de aquisição, manutenção, e acesso às informações, todas inerentes à atividade de gestão da informação e do conhecimento, tornam as ontologias extremamente relevantes nas soluções desta área".

Esse sistema atua como modelos estruturantes de informações formais e o grau dessa estruturação depende da linguagem que se é utilizada na construção e formalização do sistema construído. Cada busca realizada no sistema de recuperação de informação é estruturada por meio de uma linguagem específica que, por sua vez, contribuirá para a estruturação de uma ontologia para gestão de conhecimento jurídico.

\section{CONSIDERAÇÕES FINAIS}

Os exercícios da Organização e Representação do Conhecimento não devem ser vistos como atividades atuais para gestão do conhecimento. Esses exercícios devem ser desenvolvidos por profissionais da informação advindos da Ciência da Informação e que estejam aliados a pluralidade disciplinar que se aplicam e se desenvolvem. Dias, Almeida e Souza (2017) ressalvam a importância da atuação de pesquisadores da CI nos estudos de ontologias para representação do conhecimento, mesmo que haja resistência da área às ontologias.

Organizações e indivíduos anseiam pelo compartilhamento, decifração e uso de informações, tão logo é fundamental considerar as práticas de estudo do objeto desta pesquisa - a informação estratégica, buscando cooperar para estudos da área de Gestão, Organização e 
Representação da Informação e do Conhecimento, contribuindo principalmente para base teórica e prática de estudos para a Ciência da Informação.

Destarte, de acordo com a questão de pesquisa e o objetivo aqui proposto, foi possível compreender a contribuição do uso de ontologias na gestão do conhecimento jurídico, apontando como elemento principal as questões de competência que podem ser extraídas do sistema, além de contribuir ativamente para o processo de avaliação e melhoria do sistema e que cooperam para a eficiência do desempenho das atividades de gestão e organização da informação como aquisição, a manutenção, a representação, a busca e o acesso de informações.

\section{REFERÊNCIAS}

CARLAN, E. Sistemas de Organização do Conhecimento: uma reflexão no contexto da Ciência da Informação. 2010. 100f. Dissertação (Mestrado em Ciência da Informação) Universidade de Brasília, Brasília, 2010. Disponível em: <http://www.repositorio.unb.br/bitstream/10482/7465/1/2010_ElianaCarlan.pdf>. Acesso em: 17 abr. 2017.

CERQUEIRA, R. F. P. de. Método de modelagem domínio-ontológica do Direito Positivo Brasileiro. 2007. 131f. Dissertação (Mestrado em Ciência da Informação) - Universidade Federal de Minas Gerais, Belo Horizonte. Disponível em: <http://www.bibliotecadigital.ufmg.br/dspace/handle/1843/VALA-74QKF5>. Acesso em: 17 abr. 2017.

CONSELHO NACIONAL DE JUSTIÇA. Justiça em números. 2017. Disponível em: <http://www.cnj.jus.br/programas-e-acoes/pj-justica-em-numeros>. Acesso em: 17 abr. 2017.

DIAS, G. A.; ALMEIDA, J. F. V. R.; SOUZA, M. O Uso do Termo Ontologia em Comunicações Orais no GT-2 do ENANCIB. In: ENCONTRO NACIONAL DE PESQUISA EM CIÊNCIA DA INFORMAÇÃO, 18., 2017, Marília. Anais... Marília: ANCIB, 2017. Disponível em <http://enancib.marilia.unesp.br/index.php/xviiienancib/ENANCIB/paper/view/353>. Acesso em: 10 jan. 2018.

FUSCO, E.; MUCHERONI, M. L.; CONEGLIAN, C. S. Plataforma Informacional do Ecossistema Paulista de Inovação: Modelo Computacional e Semântico de Apoio à Inovação. In: ENCONTRO NACIONAL DE PESQUISA EM CIÊNCIA DA INFORMAÇÃO, 18., 2017, Marília. Anais... Marília: ANCIB, 2017. Disponível em: <http://enancib.marilia.unesp.br/index.php/xviiienancib/ENANCIB/paper/view/226>. Acesso em: 10 jan. 2018.

LIMA, G. A.; MACULAN, B. C. M. S. Estudo comparativo das estruturas semânticas em diferentes sistemas de organização do conhecimento. Ciência da Informação, Rio de 
? INOVAÇão

Janeiro, v. 46, n. 1, 2017. Disponível em: <http://revista.ibict.br/ciinf/article/view/4014>. Acesso em: 10 jan. 2018.

MOREIRO GONZÁLEZ, J. A. Linguagens documentárias e vocabulários semânticos para a web: elementos conceituais. Salvador: EDUFBA, 2011.

PORTER, M. E. Vantagem competitiva: criando e sustentando um desempenho superior. Rio de Janeiro: Campus, 1992.

RAMALHO, R. A. S. Desenvolvimento e utilização de ontologias em bibliotecas digitais: uma proposta de aplicação. 2010. 145f. Tese (Doutorado em Ciência da Informação) Universidade Estadual Paulista, Marília, 2010. Disponível em: <https://repositorio.unesp.br/handle/11449/103376>. Acesso em: 17 dez. 2017. 


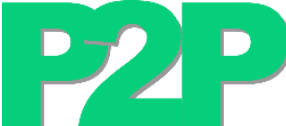

? INOVAÇão

52

P2P \& INOVAÇÃO, Rio de Janeiro, v. 4 n. 2, p.41-52, Mar./ Ago. 2018. 